\title{
BMJ Open Enhancing primary care capacity in chronic kidney disease management: a quality improvement educational initiative
}

\author{
Michelle D Smekal (10 , ${ }^{1}$ Aminu K Bello, ${ }^{2}$ Maoliosa Donald, ${ }^{1}$ Deenaz Zaidi, ${ }^{2}$ \\ Kerry McBrien, ${ }^{3}$ Katrina Nicholson, ${ }^{3}$ Ellen Novak, ${ }^{4}$ Brenda Hemmelgarn ${ }^{1,5}$
}

To cite: Smekal MD, Bello AK, Donald M, et al. Enhancing primary care capacity in chronic kidney disease management: a quality improvement educational initiative. BMJ Open 2021;11:e046068. doi:10.1136/ bmjopen-2020-046068

- Prepublication history and additional supplemental material for this paper are available online. To view these files, please visit the journal online (http://dx.doi.org/10.1136/ bmjopen-2020-046068)

Received 19 0ctober 2020 Accepted 15 October 2021

\section{Check for updates}

(C) Author(s) (or their employer(s)) 2021. Re-use permitted under CC BY-NC. No commercial re-use. See rights and permissions. Published by BMJ.

${ }^{1}$ Medicine, University of Calgary, Calgary, Alberta, Canada ${ }^{2}$ Medicine, University of Alberta, Edmonton, Alberta, Canada

${ }^{3}$ Family Medicine, University of Calgary, Calgary, Alberta, Canada

${ }^{4}$ Alberta Kidney Care, Alberta Health Services, Calgary,

Alberta, Canada

${ }^{5}$ Faculty of Medicine and Dentistry, University of Alberta, Edmonton, Alberta, Canada

Correspondence to Dr Brenda Hemmelgarn; Brenda.Hemmelgarn@ahs.ca

\section{ABSTRACT}

Background Gaps in identification, medical management and appropriate referral for patients with chronic kidney disease (CKD) are evident.

Objective We designed and implemented an interactive educational intervention (accredited workshop) to improve primary care providers' awareness of tools to support guideline-concordant CKD management.

Design We used the Kern method to design the educational intervention and targeted the accredited workshops to primary care team members (physicians, nurses and allied health) in Alberta, Canada. We conducted anonymous pre-workshop and post-workshop surveys to identify practice-specific barriers to care, identify potential solutions, and evaluate provider confidence pre-intervention and post-intervention. We used nonparametric statistics to analyse Likert-type survey data and descriptive content analysis to categorise responses to open-ended survey questions.

Results We delivered 12 workshops to 114 providers from September 2017 through March 2019. Significant improvements $(p<0.001)$ in confidence to appropriately identify, manage and refer patients with CKD were observed. Participants identified several patient-level, provider-level, and system-level barriers and potential solutions to care for patients with CKD; the majority of these barriers were addressed in the interactive workshop. Conclusions The Kern model was an effective methodology to design and implement an educational intervention to improve providers' confidence in managing patients with CKD in primary care. Future research is needed to determine if these perceived knowledge and confidence improvements affect patient outcomes and whether improvements are sustained long term.

\section{INTRODUCTION}

Chronic kidney disease (CKD) affects approximately $11 \%$ of adults in Canada and is associated with adverse clinical outcomes, poor quality of life and high healthcare costs. ${ }^{1}$ The majority $(>90 \%)$ of patients with CKD are managed by primary care providers (PCPs) in the community. ${ }^{2}$ Despite therapies proven to reduce the adverse consequences associated

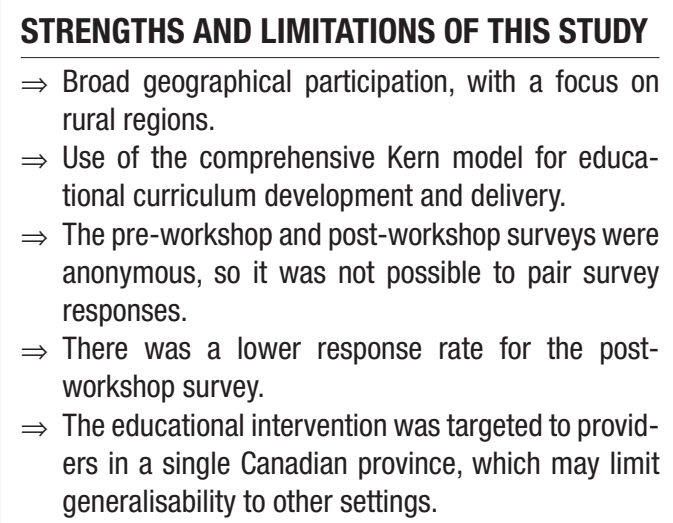

with CKD, significant gaps in delivering optimal care still exist. ${ }^{3}$ For instance, literature would suggest that $\sim 50 \%$ of older adults with CKD are not on guideline-recommended cardioprotective medications, and only $20 \%$ who meet the criteria to see a nephrologist are referred. ${ }^{4}$ Moreover, less than $20 \%$ of patients in Canada received a urine albumincreatinine test within the recommended time frame following CKD diagnosis. ${ }^{5}$ PCPs have identified several barriers to optimal care delivery for patients with CKD, including limited awareness regarding referral criteria for specialist care, absence of readily available guidelines at point of care and uncertainty about when to test for proteinuria (a key prognostic marker for kidney and cardiovascular risk). ${ }^{6}$

In an effort to improve care for patients with CKD in Alberta, Canada, several resources were developed and tailored for PCPs to facilitate guideline-concordant care for this patient group, with the aim of improving early identification and appropriate management as well as timely referral to specialists when indicated. These tools include an online clinical pathway for CKD (www.ckdpathway.ca), a provincial electronic specialist referral and 
advice portal, and patient management tools embedded within the predominant primary care electronic medical record (EMR) platform. ${ }^{7-9}$ In this quality improvement initiative, we aimed to develop and implement an interactive educational intervention (accredited workshop) to improve the capacity (knowledge and awareness) of CKD management in primary care.

\section{METHODS}

\section{Setting and participants}

Healthcare in Canada is provided through a singlepayer, universal system that provides comprehensive coverage, with no out-of-pocket cost, for physician and hospital services to all residents. ${ }^{10}$ In Alberta, healthcare delivery is organised by a single provincial body, Alberta Health, through five geographical zones (South, Central, Calgary, Edmonton and North) (online supplemental file 1). Several province-wide Strategic Clinical Networks (SCNs) were created by Alberta Health Services in 2012 to implement and coordinate novel solutions to improve care for Albertans; while much of this work is hospital based, the SCNs also work with PCPs, through the Primary Health Integration Network to improve primary-secondary care integration. ${ }^{11}$ Primary Care Networks (PCNs) operate within the province with an aim to provide care following a team-based model (including physicians, nurses and allied health providers). Currently, approximately 3700 PCPs provide care through 41 PCNs in Alberta. ${ }^{12}{ }^{13}$ Most patients with
CKD are cared for in primary care settings in Alberta $(>90 \%),{ }^{2}$ therefore the educational intervention was targeted to primary care team members.

\section{Interactive educational intervention}

We developed and delivered an interactive continuing medical education (CME) programme through a series of workshops across Alberta. This was designed to meet the identified needs of PCPs for CKD management, and improve guideline-concordant care for patients with CKD in primary care settings. We used the six-step Kern model to guide educational content development and delivery (figure 1). ${ }^{14}$ The Kern model, developed at the Johns Hopkins University School of Medicine, is a learnercentred systematic approach that explicitly links curriculum to identified healthcare needs; each of the six steps reinforces each other in a cycle and can be used to inform continuous curriculum improvement. ${ }^{14}$ The Kern model was developed specifically for medical education and has been applied successfully in a number of settings for over 20 years. ${ }^{1415}$ It has been widely applied to evaluate knowledge gaps and needs assessments for educational interventions. The model was selected to guide curriculum development and delivery in this project as it incorporates many components shown to positively impact clinical practice following CME activities. It afforded opportunities for highly interactive sessions using multiple teaching methods/exposures, and is based on learner-focused needs and outcomes. ${ }^{16}$

Step 1. Problem Identification

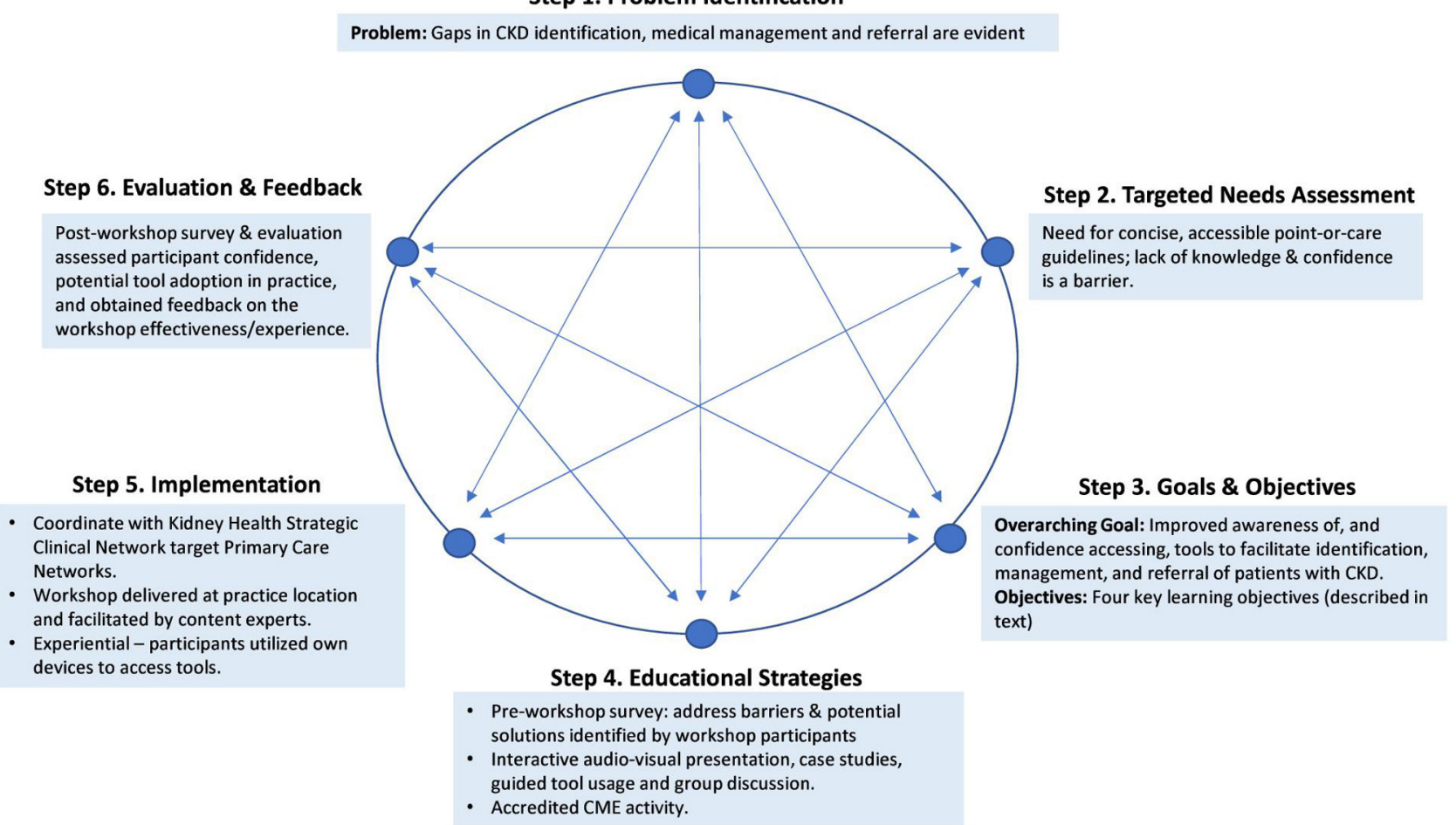

Figure 1 Application of Kern's six-step model to curriculum development and implementation for this intervention. CKD, chronic kidney disease; CME, continuing medical education. 


\section{Workshop development}

Workshop development encompassed the first three steps of the Kern model:

Step 1: Problem Identification and General Needs Assessment. Despite availability of clinical practice guidelines ${ }^{17-19}$ there remain gaps in CKD identification, medical management and referral for patients with CKD treated in primary care environments in Alberta. ${ }^{35}$

Step 2: Targeted Needs Assessment. Previous work identified insufficient access to concise guidelines and lack of confidence by providers to care for patients with CKD as leading barriers to appropriate care ${ }^{68}$ and identified an online clinical pathway as a desired tool to improve guideline uptake $;^{20}$ consequently, the CKD clinical pathway (CKD-P) (www.ckdpathway.ca) was designed and implemented in 2014 to support guideline-concordant care. ${ }^{21}$ One of the needs stemming from this work was a desire for continued dissemination of the CKD-P and related tools, in primary care environments.

Step 3: Goals and Objectives. The educational workshop was designed to align with the Royal College of Physicians and Surgeons of Canada CanMEDS Physician Competency Framework. ${ }^{22}$ The framework encompasses a set of thematic roles physicians require to effectively meet the healthcare needs of the people they serve. ${ }^{22}$ At the completion of the workshop, PCPs would have greater awareness of, and confidence accessing, tools to facilitate appropriate identification, guideline-concordant medical management and timely referral of patients with CKD.

The key learning objectives were:

1. Access the online CKD-P $\mathrm{P}^{8}$ and identify, medically manage, and appropriately refer patients with $\mathrm{CKD}$, based on evidence-based guidelines. ${ }^{17}$

2. Access the Nephrology eReferral system through the Alberta Health Services Netcare portal and understand when to initiate a referral or specialist advice request and how to complete one. ${ }^{723}$

3. Use EMR database query features, such as a complex disease management dashboard, to proactively identify and recall patients with CKD and facilitate ongoing monitoring using the Comprehensive Annual Care Plan (CACP) (the CACP is a provincially developed tool used to support the care of patients with specific chronic diseases, including $\mathrm{CKD}$, using a formal care plan).

4. Access and use an enhanced CACP template, with embedded clinical decision support, to streamline workflow and ensure guideline-concordant care delivery for patients with CKD.

\section{Pre-workshop survey and workshop implementation}

This phase encompassed steps 4-5 of the Kern model:

Step 4: Educational Strategies. The curriculum included a pre-workshop survey and a 1-hour interactive workshop:

1. Pre-workshop survey: the pre-workshop survey was sent to workshop participants via email link approximately 1 week prior to the workshop (online supplemental file 2). The purpose was to assess participants' confidence and barriers and facilitators to caring for patients with CKD using a combination of Likert and open-ended response questions. The survey questions were derived directly from the clinical practice gaps and targeted needs assessment identified in steps 1 and 2 of the model. The purpose of this survey was to encourage individual reflection and to identify unique barriers in the participants' clinical setting that could be addressed during the workshop, in addition to the planned curriculum. The survey results and barriers were aggregated for each practice group and presented during the workshop, which allowed for targeted discussion.

2. Interactive workshop: the interactive educational intervention was facilitated by a nephrologist $(\mathrm{BH})$ or primary care physician (DZ) with expertise in nephrology. The facilitator used several techniques to engage learners, including an interactive audio-visual presentation, case studies, guided tool usage and group discussion. Workshop content was developed and reviewed by two nephrologists $(\mathrm{BH}, \mathrm{AKB})$, three primary care physicians (DZ, KM, TN) and one nurse practitioner (EN) with expertise in nephrology. The educational programme was accredited by the College of Family Physicians of Canada for up to 2.0 MAINPRO + continuing educational credits.

Step 5: Implementation. The accredited education sessions were advertised by the Kidney Health SCN from September 2017 through March 2019. The workshop advertisements were targeted to PCPs throughout the province with an emphasis on rural areas, where barriers to care are heightened and clinical outcomes are poorer compared with urban regions. ${ }^{524} 25$ Workshops were primarily delivered in person at or near providers' clinic locations, with one workshop delivered via an interactive video-conference format. Due to the experiential nature of the workshops, that required direct access to relevant online tools, attendees were asked to bring a laptop or tablet that could connect to these tools to fully participate in the workshop.

\section{Post-workshop survey and evaluation}

Step 6: Evaluation and Feedback. A post-workshop survey was implemented to assess participants' confidence and evaluate workshop effectiveness. The survey used questions from the pre-workshop survey and also asked participants how likely they would be to use the tools accessed during the workshop in their routine clinical practice (online supplemental file 3). The survey included a combination of Likert and open-ended response questions and was initially collected via an online link, which was transitioned to paper surveys immediately following the workshop to increase response rates.

\section{Analysis}

Quantitative data analysis

Non-parametric statistics were used for the Likerttype survey data; ${ }^{26}$ pre-implementation and 
post-implementation differences in survey responses were compared using the Wilcoxon Mann-Whitney rank-sum test for unmatched data. ${ }^{27}{ }^{28}$ Analyses were performed using Stata V.14 (StataCorp, College Station, Texas, USA).

\section{Qualitative data analysis}

We used a descriptive content analysis approach to identify categories and summarise responses to open-ended questions. ${ }^{29} 30$ Descriptive content analysis approaches are used to describe and categorise text data and identify common trends, but with a low-level of interpretation (that is, the analysis stays 'close to the data'); consequently, we did not use theory to guide analysis of barriers and facilitators to CKD care. ${ }^{30} 31$ Text data were imported into NVivo V.12 software to facilitate data analysis (QSR International, Doncaster, Australia). One investigator (MDS) with expertise in qualitative analysis reviewed the text responses to open-ended questions, categorised the response data, and discussed the raw and categorised data with two investigators (BH, MD) to ensure completeness and achieve consensus on the final categories and interpretation.

\section{Patient and public involvement}

Because this was an accredited continuing medical education workshop targeted to PCPs and developed by content experts, we did not consult with patients or the public in the design, conduct or dissemination of this initiative.

\section{RESULTS}

Twelve accredited workshops were delivered to 114 participants throughout Alberta from September 2017 through March 2019. Overall, 32\% of workshop participants were from the North zone, $24 \%$ from the Central zone, $22 \%$ from the Edmonton zone, $16 \%$ from the South zone and $7 \%$ from the Calgary zone (). Over $70 \%$ of workshop participants were practising in rural locations at the time of the workshop. Approximately $76 \%$ of participants completed the pre-workshop survey and $42 \%$ completed the post-workshop survey. The majority of survey participants were physicians (63\% pre; $67 \%$ post) followed by nurses/nurse practitioners (16\% pre; $17 \%$ post) (table 1$)$.

Of those who completed the pre-workshop survey, $79 \%$ had previously heard about the online CKD-P, although only $37 \%$ had used the tool. Overall, $62 \%$ had heard of the eReferral portal and $9 \%$ had used the tool, while $25 \%$ had used dashboard features in their EMR to proactively identify and manage patients with CKD.

\section{Qualitative results}

In the pre-workshop survey, participants were asked to list key risk factors and barriers to management of patients with CKD in primary care. In the pre-workshop survey, participants also identified a number of patient-level,
Table 1 Participant roles and workshop locations

\begin{tabular}{|c|c|c|}
\hline & $\begin{array}{l}\text { Pre-survey } \\
\text { n (\%) }\end{array}$ & $\begin{array}{l}\text { Post-survey } \\
\text { n (\%) }\end{array}$ \\
\hline \multicolumn{3}{|l|}{ Clinic role } \\
\hline Clinic staff & $7(7.9)$ & $4(8.3)$ \\
\hline Dietitian & $6(6.7)$ & $1(2.1)$ \\
\hline Pharmacist & $4(4.5)$ & $2(4.2)$ \\
\hline Exercise specialist & $1(1.1)$ & $0(0)$ \\
\hline Nurse & $10(11.2)$ & $5(10.4)$ \\
\hline Nurse practitioner & $4(4.5)$ & $3(6.3)$ \\
\hline Physician assistant & $1(1.1)$ & $0(0)$ \\
\hline Physician & $56(62.9)$ & $32(66.7)$ \\
\hline Other & $0(0)$ & $1(2.1)$ \\
\hline Total & $89(100)$ & $48(100)$ \\
\hline \multicolumn{3}{|c|}{ Workshop clinic locations by AHS zone } \\
\hline North & $32.30 \%$ & \\
\hline Edmonton & $21.50 \%$ & \\
\hline Central & $23.70 \%$ & \\
\hline Calgary & $6.50 \%$ & \\
\hline South & $16.10 \%$ & \\
\hline
\end{tabular}

AHS, Alberta Health Services.

provider-level, and system-level challenges and potential solutions to management in a primary care setting; the relationships between workshop educational components and these challenges and proposed solutions are depicted in figure 2. Participant responses to practicespecific challenges and potential solutions are listed in online supplemental file 4.

\section{Patient-level}

Workshop participants identified several important patient-level barriers that they perceived may impact optimal care delivery. The most common patient-level barriers identified included travel distance, self-efficacy and following recommended treatments, and overall health literacy. Workshop participants identified a number of strategies to help overcome these barriers, including a patient-centred approach, education and goal setting, regular follow-up, help for changes in lifestyle (such as weight loss programmes and diabetes management support), and financial support for medications. For example, one nurse suggested they 'try to set small achievable goals with patient[s] to make a healthy change' and another suggested 'patient education, goal setting, regular follow up [and] increased financial support for medications would be beneficial' for patients with CKD. Existing programmes to support patients with complex health needs were also suggested to be beneficial: one physician noted that a 'locally run diabetic nephropathy prevention clinic has been super helpful' and another recommended an 'expansion of service provided by [the] diabetic 


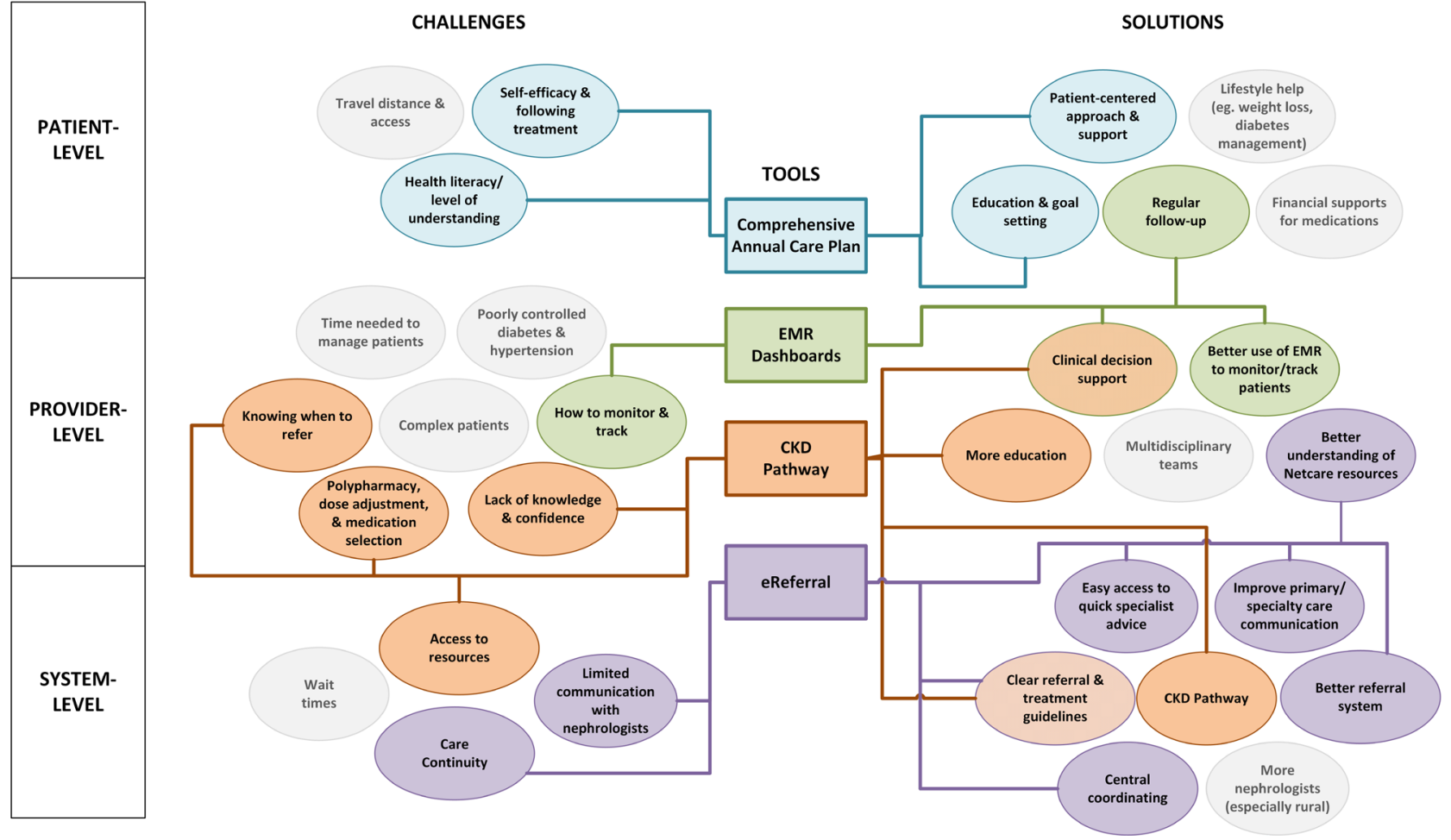

Figure 2 Relationship between workshop educational components/tools and CKD management challenges and potential solutions identified by workshop participants prior to educational intervention. Grey boxes denote challenges and solutions that are unrelated to the workshop educational components. CKD, chronic kidney disease; EMR, electronic medical record.

nephropathy prevention clinic to become more of a nephropathy prevention clinic (include non-diabetics with nephropathy as well as diabetics who do not yet have nephropathy)'. With respect to travel distance, some participants also indicated that use of telehealth has helped to overcome access-related challenges: 'More use is now being made of telemedicine, the closest actual out reach clinic is $1.5 \mathrm{hrs}$ away, which is a huge issue for the commonly elderly and debilitated patients' (physician).

\section{Provider-level}

The most common provider-level barriers included a perceived lack of knowledge and confidence to manage patients with CKD in general; limited awareness about indications for referral to nephrology; complexity with prescribing medicines in the context of multimorbidity and low kidney function, poorly controlled diabetes and hypertension; and the time needed to care for patients adequately. The primary solutions included the need for more education for providers: 'participate in educational opportunities' (nurse practitioner), 'more education regarding $[\mathrm{CKD}]$ and a resource to refer to as needed' (pharmacist), 'more education/training' (physician) and a belief that the workshop would be helpful: '[I] anticipate this educational session will be very helpful' (physician). Other potential solutions identified by participants included clinical decision support, more effective use of EMRs to proactively monitor and track patients, incorporating a multidisciplinary team approach to care, and developing a better awareness and understanding of the resources and tools available through Alberta Health Services' Netcare portal. For example, some participants indicated that 'utilization of reminders for patients to follow-up' (physician) and 'setting a pop up in the EMR to remind me to consider renal clearance when writing any prescription for a patient' (physician) would be helpful while others suggested that 'understanding the CKD pathway and system, and better utilizing the resources available on Netcare' (pharmacist) and 'learn[ing] more about the Netcare eReferral and eAdvice' portals would be helpful (physician).

\section{System-level}

The primary system-level challenges identified by participants include limited communication with nephrologists, care continuity, access to resources (including issues accessing wi-fi-dependent portals in rural settings) and specialist wait times. Participants identified several potential solutions to overcome these challenges, such as 'better online guidelines about CKD and comorbidities' (physician), 'clear guidelines for referrals' (dietitian), 'more guidance from specialists' (physician) and 'facilitating communication lines between specialist clinics and PCN clinic staff' (dietitian), 'getting advice faster' (physician), and an ability to obtain 'quick access to nephro[logy] for non-urgent advice'. Other potential solutions included central coordinating, a better referral system and 'more nephrologists in the country' (physician), especially in rural locations. 
Quantitative results

Of the common risk factors for $\mathrm{CKD}^{32}$ identified in the pre-workshop survey, $91 \%$ of participants identified diabetes, $48 \%$ identified hypertension and cardiovascular disease, $10 \%$ identified older age and less than $5 \%$ identified other risk factors, such as acute kidney injury, nephrotoxins, family history, smoking, obesity and ethnicity. Participants were also asked to rate their comfort level in four areas relating to knowledge and confidence managing patients with CKD in both the pre-workshop and post-workshop surveys: (1) identifying a patient with CKD; (2) administering guideline-concordant medication therapies for patients with CKD; (3) knowing when a referral to a nephrologist is indicated; and (4) providing resources and suggesting guideline-recommended goals for patients with CKD. When comparing the presurvey and post-survey responses, there were significant improvements $(\mathrm{p}<0.001)$ in the comfort level reported by all participants (for all four areas measured) in the post-workshop survey (figure 3A) and these significant differences were retained when analysing physician-only responses (figure $3 \mathrm{~B}$ ).

Following the workshop, the majority of participants indicated that they planned to use the tools introduced in the educational session; approximately $90 \%$ indicated they planned to use the CKD-P and approximately $70 \%$ planned to use the eReferral portal to either refer patients to nephrology or submit an electronic specialist advice request. In the workshop evaluation, over $90 \%$ of participants agreed that the content was relevant, the session enhanced their knowledge and that they intend to use the information learnt in their primary care practice (online supplemental file 5).

A) Workshop participants comfort level, All respondents (pre $n=89$, post $n=48$ )

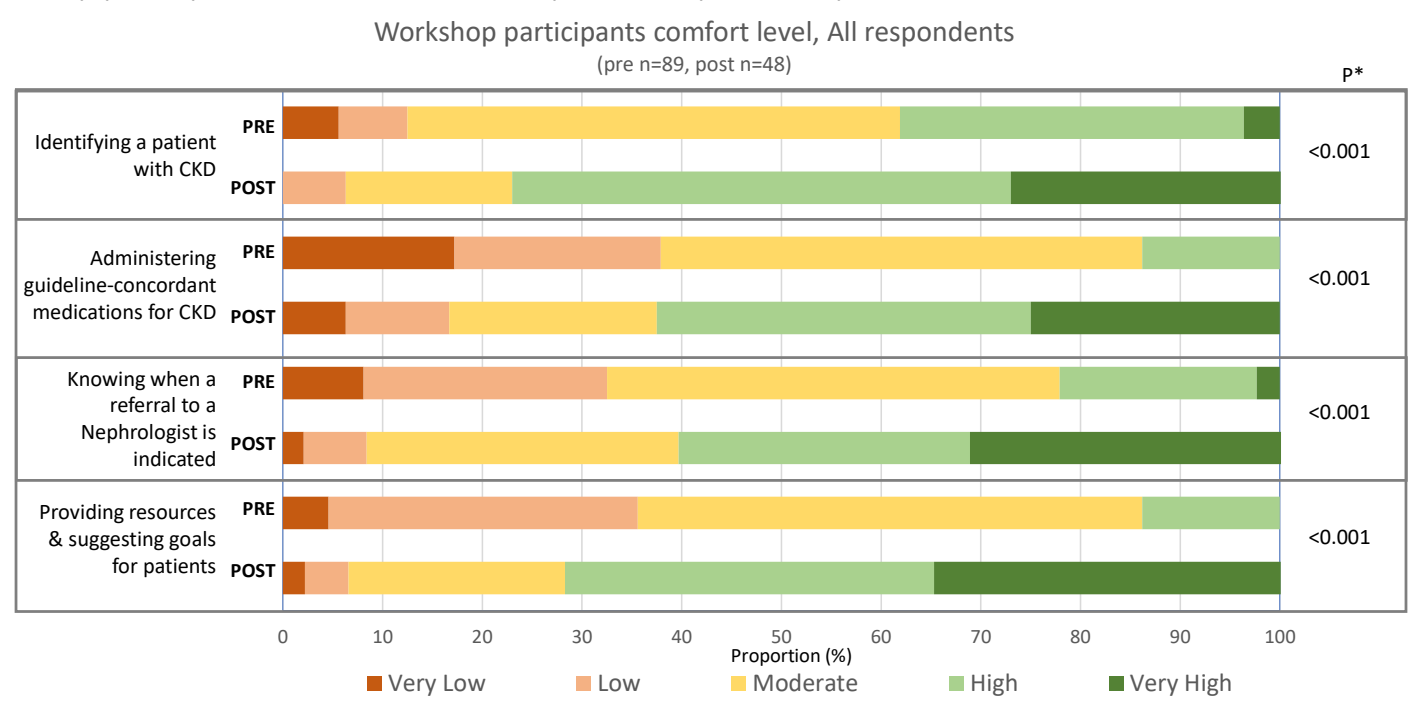

B) Workshop participants comfort level, Physicians only (pre $n=56$, post $n=32$ )

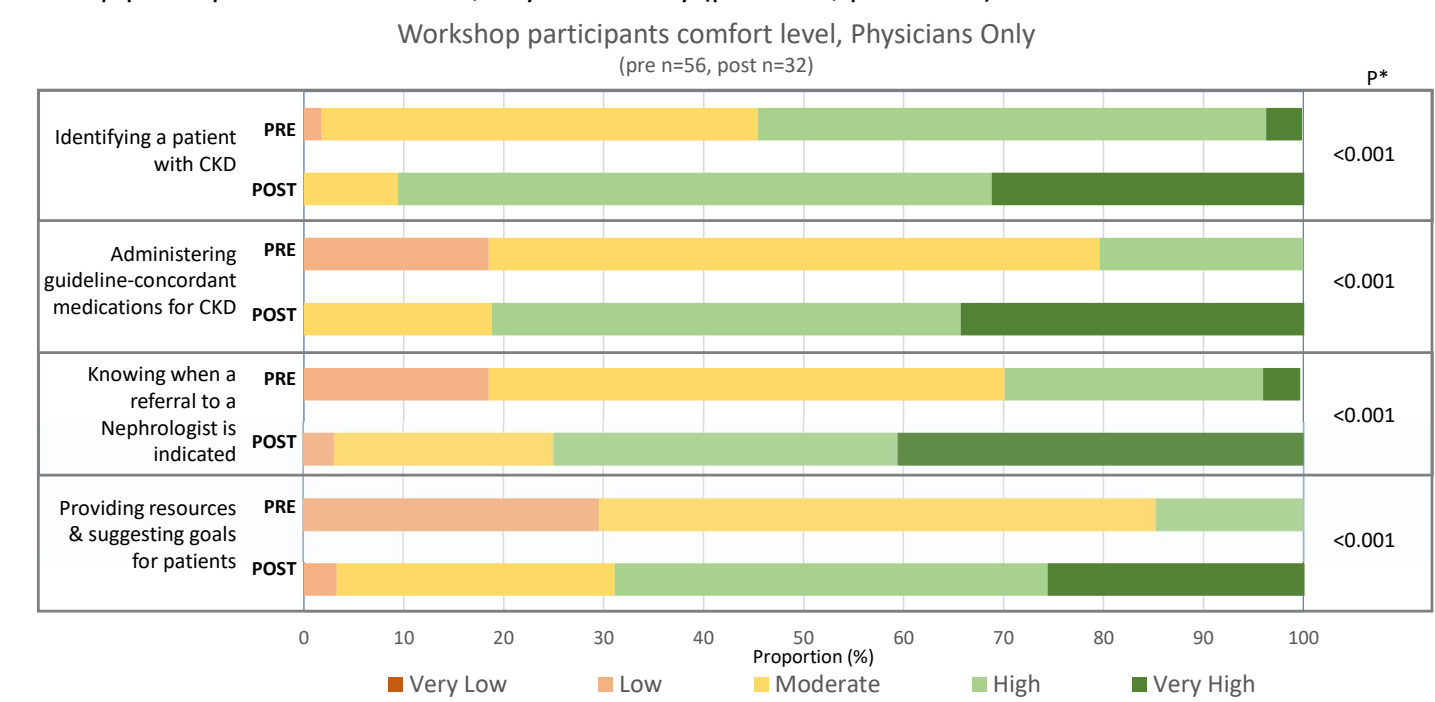

Figure 3 Pre/post-survey responses for (A) all workshop participants and (B) physician participants only. CKD, chronic kidney disease. 


\section{DISCUSSION}

The aim of this quality improvement study was to design and implement an educational intervention to address important gaps in CKD care and improve healthcare providers' perceived knowledge and confidence to manage patients with CKD in primary care. Prior to participating in the interactive workshop, participants identified a number of patient-level, provider-level and system-level challenges, and potential solutions to managing CKD in the primary care setting. Following the educational intervention, participants indicated that the workshop was relevant to their practice, and significant improvements in knowledge and confidence to identify, treat, and refer patients with CKD were observed.

Pre-workshop survey results identified a gap in the awareness of CKD risk factors among participants. While diabetes was commonly identified as a significant risk factor for CKD, other important risk factors, including hypertension, cardiovascular disease, advanced age, nephrotoxins (such as non-steroidal anti-inflammatory drugs), family history and previous acute kidney injury, were not well recognised, suggesting an important knowledge gap. This is especially relevant considering that the early stages of CKD are commonly 'silent' and early diagnosis, which is dependent on laboratory testing, is key to initiating kidney protective therapies and preventing disease progression. ${ }^{35}$

Our study also identified several important patientlevel, provider-level and system-level challenges to CKD management in primary care. Many of these challenges are at least partially remedied by tools that are available to primary care physicians in Alberta, Canada (such as the CKD-P, eReferral portal, CACP and EMR dashboards), and were introduced to participants during the educational intervention. Many participants were aware of these tools, but had not used them in clinical practice, suggesting that a concerted effort to disseminate these tools is needed to improve awareness and uptake in primary care. Several challenges identified by participants were beyond the scope of this education intervention, such as travel distance and access for patients, time constraints, specialty wait times, internet wi-fi reliability in rural locations and financial support for medications. These system-level challenges deserve further attention and may serve to better support CKD management in primary care in Alberta. Many of these challenges have also been reported in the literature..$^{52433-35}$ The time required to care for patients with chronic conditions is a significant challenge in primary care, ${ }^{33}$ and the density and ratio of nephrologists to patients (14:1000) in Canada is one of the lowest in the Organisation for Economic Co-operation and Development, ${ }^{24}{ }^{36}$ though it is not clear how this low ratio may, or may not, affect patient outcomes. ${ }^{37}$ Additionally, inadequate financial support for medications has been reported as a common reason for poor medication adherence, poorer clinical outcomes and increased healthcare utilisation over the long term. ${ }^{38-40}$ As clinical tools designed to support PCPs are increasingly dependent on internet wi-fi access, the lack of infrastructure in rural and remote regions presents a significant barrier to PCPs' uptake of these tools. ${ }^{41}$

We used an interactive small-group educational intervention to improve PCPs' knowledge and confidence in managing CKD patient care in a community setting. Educational outreach has been reported to be an effective knowledge translation methodology for healthcare provider audiences ${ }^{16} 42$ and our previous research suggests that small-group in-person activities are effective means for increasing the use of an online clinical pathway in primary care. ${ }^{43}$ By using the structured Kern model to design the educational intervention, we were able to adapt content to the local, primary care context and target three identified knowledge gaps: early identification of CKD, administration of guideline-concordant drug therapies and identifying when referral to a nephrologist is indicated. This approach was ultimately successful, with significant improvements evident in the pre/post-survey, although it is unclear if this new knowledge was sustained or translated into practice changes as the study did not evaluate the effectiveness of the curriculum in enhancing CKD management beyond participants' participation in the continuing medical education session.

Strengths of this study include broad geographical participation across the province of Alberta, with a focus on rural regions, and use of a comprehensive model for educational curriculum development and delivery. However, there are limitations that should be recognised when interpreting the results. The pre-workshop and post-workshop surveys were anonymous, so it was not possible to pair survey responses. There was also a lower response rate for the post-workshop survey, however, the response rate improved when the administration method was modified to a paper-based survey, rather than electronic, following the workshop. It is also important to note that we used qualitative descriptive methodology, specifically conventional content analysis, to summarise perceived barriers and facilitators to CKD care; we did not use theory to guide analysis. Finally, the educational intervention was targeted to providers in a single Canadian province, which may limit generalisability to other settings.

In summary, we implemented a robust quality improvement-based educational intervention, using the Kern model to improve the capacity of PCPs in the management of CKD. Educational interventions leveraging this approach may effectively improve knowledge and confidence among PCPs and improve clinical care for patients with CKD. More research is needed to understand if these knowledge improvements affect clinical practice, and whether improvements are sustained long term.

Twitter Michelle D Smekal @msmekal

Acknowledgements We would like to acknowledge the Kidney Health Strategic Clinical Network (SCN) for their support with advertising and organising workshops and administering pre-workshop and post-workshop surveys. 
Contributors All authors in this study have contributed to this manuscript and approve of this submission. MDS, AKB, DZ, KM, KN, EN and BH contributed to the educational initiative design and delivery. MDS, AKB, MD and BH drafted the article. All authors contributed to the quality improvement project and provided critical revisions to this manuscript. BH is the guarantor for the study.

Funding This study was supported by the Canadian Institutes of Health Research (CIHR; grant number 10021854). BH is supported by the Roy and Vi Baay Chair in Kidney Research.

Competing interests None declared.

Patient consent for publication Not required.

Ethics approval Not applicable. This educational initiative was reviewed by the University of Calgary Conjoint Health Research Ethics Board and deemed a quality improvement project. Data were collected as a required component of the College of Family Physicians of Canada MAINPRO + continuing education accreditation for this educational activity.

Provenance and peer review Not commissioned; externally peer reviewed.

Data availability statement Data are available upon reasonable request. All data relevant to the study are included in the article or uploaded as supplemental information. The datasets used and/or analyzed during the current study are available from the corresponding author on reasonable request.

Supplemental material This content has been supplied by the author(s). It has not been vetted by BMJ Publishing Group Limited (BMJ) and may not have been peer-reviewed. Any opinions or recommendations discussed are solely those of the author(s) and are not endorsed by BMJ. BMJ disclaims all liability and responsibility arising from any reliance placed on the content. Where the content includes any translated material, BMJ does not warrant the accuracy and reliability of the translations (including but not limited to local regulations, clinical guidelines, terminology, drug names and drug dosages), and is not responsible for any error and/or omissions arising from translation and adaptation or otherwise.

Open access This is an open access article distributed in accordance with the Creative Commons Attribution Non Commercial (CC BY-NC 4.0) license, which permits others to distribute, remix, adapt, build upon this work non-commercially, and license their derivative works on different terms, provided the original work is properly cited, appropriate credit is given, any changes made indicated, and the use is non-commercial. See: http://creativecommons.org/licenses/by-nc/4.0/.

ORCID iD

Michelle D Smekal http://orcid.org/0000-0003-0960-3628

\section{REFERENCES}

1 Arora P, Vasa P, Brenner D, et al. Prevalence estimates of chronic kidney disease in Canada: results of a nationally representative survey. CMAJ 2013;185:423 doi:10.1503/cmaj.120833

2 Manns B, Tonelli M, Culleton B, et al. A cluster randomized trial of an enhanced eGFR prompt in chronic kidney disease. Clin J Am Soc Nephrol 2012;7:565-72.

3 James MT, Hemmelgarn BR, Tonelli M. Early recognition and prevention of chronic kidney disease. Lancet 2010;375:1296-309. doi:10.1016/S0140-6736(09)62004-3

4 Alberta Kidney Disease Network. Prevalence of severe kidney disease and use of dialysis and transplantation across Alberta from 2004-2013 Alberta annual kidney care report kidney-strategic clinical network executive summary background 2015.

5 Bello AK, Ronksley PE, Tangri N, et al. Quality of chronic kidney disease management in Canadian primary care. JAMA Netw Open 2019;2:e1910704. doi:10.1001/jamanetworkopen.2019.10704

6 Hemmelgarn BR, Manns BJ, Straus S, et al. Knowledge translation for nephrologists: strategies for improving the identification of patients with proteinuria. J Nephrol 2012;25:933-43.

7 Bello AK, Molzahn AE, Girard LP, et al. Patient and provider perspectives on the design and implementation of an electronic consultation system for kidney care delivery in Canada: a focus group study. BMJ Open 2017;7:e014784.

8 Donald M, McBrien K, Jackson W, et al. Development and implementation of an online clinical pathway for adult chronic kidney disease in primary care: a mixed methods study. BMC Med Inform Decis Mak 2016;16 doi:10.1186/s12911-016-0350-z

9 Al Hamarneh YN, Hemmelgarn B, Curtis C, et al. Community pharmacist targeted screening for chronic kidney disease. Can Pharm J 2016;149:13-17.
10 Martin D, Miller AP, Quesnel-Vallée A, et al. Canada's universal health-care system: achieving its potential. Lancet 2018;391-1718-35. doi:10.1016/S0140-6736(18)30181-8

11 Yiu V, Belanger F, Todd K. Alberta's strategic clinical networks: enabling health system innovation and improvement. CMAJ 2019;191:S1-3. doi:10.1503/cmaj.191232

12 Cunningham CT, Seidel J, Bahler B, et al. Primary health care integration network: building bridges in Alberta's health system. CMAJ 2019;191:S47-8. doi:10.1503/cmaj.190595

13 Manns BJ, Tonelli M, Zhang J, et al. Enrolment in primary care networks: impact on outcomes and processes of care for patients with diabetes. CMAJ 2012;184:E144-52.

14 Kern DE, Thomas PA, Patricia A, et al. Curriculum development for medical education: a six-step approach. Johns Hopkins University Press, 2009.

15 Kern DE, Bass EB, Thomas PA. Curriculum development for medical education: a six step approach. Johns Hopkins University Press, 1998

16 Cervero RM, Gaines JK. The impact of CME on physician performance and patient health outcomes: an updated synthesis of systematic reviews. J Contin Educ Health Prof 2015;35:131-8. doi:10.1002/chp.21290

17 Guidelines - KDIGO [online]. Available: https://kdigo.org/guidelines/ [Accessed 15 Jun 2020].

18 Diabetes Canada clinical Practice Guidelines Expert Committee. Diabetes Canada 2018 clinical practice guidelines for the prevention and management of diabetes in Canada. Can J Diabetes 2018;42.

19 Leung AA, Daskalopoulou SS, Dasgupta K, et al. Hypertension Canada's 2017 guidelines for diagnosis, risk assessment, prevention, and treatment of hypertension in adults. Can J Cardiol 2017;33:557-76. doi:10.1016/j.cjca.2017.03.005

20 Hemmelgarn BR, Manns BJ, Straus S, et al. Knowledge translation for nephrologists: strategies for improving the identification of patients with proteinuria. J Nephrol 2012;25:933-43. doi:10.5301/ jn.5000226

21 Donald M, Mcbrien K, Jackson W. Development and implementation of an online clinical pathway for adult chronic kidney disease in primary care: a mixed methods study, 2016. Available: www. ckdpathway.ca [Accessed 9 Sep 2020].

22 Frank JR, Snell L, Sherbino J. Royal College of physicians and surgeons of Canada. CanMEDS 2015 physician competency framework. $35 \mathrm{P}$.

23 Alberta Health Services. eReferral nephrology advice Bulletin, October 20, 2016, 2015. Available: http://repositorio.unan.edu.ni/ 2986/1/5624.pdf [Accessed 15 Jun 2020].

24 Lunney M, Samimi A, Osman MA, et al. Capacity of kidney care in Canada: identifying barriers and opportunities. Can J Kidney Health Dis 2019;6:205435811987054.

25 Rucker D, Hemmelgarn BR, Lin M, et al. Quality of care and mortality are worse in chronic kidney disease patients living in remote areas. Kidney Int 2011;79:210-7. doi:10.1038/ki.2010.376

26 Sullivan GM, Artino AR. Analyzing and interpreting data from likerttype scales. J Grad Med Educ 2013:5:541-2.

27 Mann HB, Whitney DR. On a test of whether one of two random variables is stochastically larger than the other. Ann Math Statist 1947;18:50-60.

28 Wilcoxon F. Individual comparisons by ranking methods. Biometrics Bulletin 1945;1:80. doi:10.2307/3001968

29 Hsieh H-F, Shannon SE. Three approaches to qualitative content analysis. Qual Health Res 2005;15:1277-88.

30 Sandelowski M. Whatever happened to qualitative description? Res Nurs Health 2000;23:334-40.

31 Vaismoradi M, Turunen $\mathrm{H}$, Bondas T. Content analysis and thematic analysis: implications for conducting a qualitative descriptive study. Nurs Health Sci 2013;15:398-405.

32 Kazancioğlu R. Risk factors for chronic kidney disease: an update. Kidney Int Suppl 2013;3:368-71.

33 Østbye T, Yarnall KSH, Krause KM, et al. Is there time for management of patients with chronic diseases in primary care? Ann Fam Med 2005;3:209-14. doi:10.1370/afm.310

34 Junaid Nazar CM, Kindratt TB, Ahmad SMA. Barriers to the successful practice of chronic kidney diseases at the primary health care level; a systematic review. J Ren Inj Prev 2014;3.

35 Neale EP, Middleton J, Lambert K. Barriers and enablers to detection and management of chronic kidney disease in primary healthcare: a systematic review. BMC Nephrol 2020;21:83. doi:10.1186/s12882020-01731-x

36 Sharif MU, Elsayed ME, Stack AG. The global nephrology workforce: emerging threats and potential solutions! Clin Kidney J 2016;9:11-22. doi:10.1093/ckj/sfv111 
$37 \mathrm{Ku}$ E, Johansen KL, Portale AA, et al. State level variations in nephrology workforce and timing and incidence of dialysis in the United States among children and adults: a retrospective cohort study. BMC Nephrol 2015;16:2. doi:10.1186/1471-2369-16-2

38 Campbell DJT, Manns BJ, Weaver RG, et al. Financial barriers and adverse clinical outcomes among patients with cardiovascularrelated chronic diseases: a cohort study. BMC Med 2017;15:33. doi:10.1186/s12916-017-0788-6

39 Gagnon M-A. The role and impact of cost-sharing mechanisms for prescription drug coverage. CMAJ 2017;189:E680-1. doi:10.1503/ cmaj.170169
40 Law MR, Cheng L, Dhalla IA, et al. The effect of cost on adherence to prescription medications in Canada. CMAJ 2012;184:297-302. doi:10.1503/cmaj.111270

41 Goodridge D, Marciniuk D. Rural and remote care. Chron Respir Dis 2016;13:192-203. doi:10.1177/1479972316633414

42 Lau R, Stevenson F, Ong BN, et al. Achieving change in primary care-effectiveness of strategies for improving implementation of complex interventions: systematic review of reviews. BMJ Open 2015;5:e009993. doi:10.1136/bmjopen-2015-009993

43 Chong C, Smekal M, Donald M. Using Google analytics to describe dissemination strategies and utilization of an online chronic kidney disease clinical pathway tool. CSN ePoster Library 2020;2020. 